\title{
Adrenal Leiomyoma Mimicking Adrenal Malignancy: Diagnostic Challenges and Review of Literature
}

\author{
Dayanidhi Meher ${ }^{a}$, Deep Dutta ${ }^{\text {b, e }}$, Ranjana Giric ${ }^{c}$, Madhabananda Kar ${ }^{\mathrm{d}}$
}

\begin{abstract}
Leiomyoma arising from adrenals are extremely rare with less than 20 cases reported in medical literature. Leiomyomas, though benign lesions, may frequently be confused with a malignancy, both clinically and on imaging studies, especially when they occur at atypical sites like the adrenals. We present a 42 -year-old lady with cachetic symptoms, abdominal swelling and dragging sensation for 6 months, having a $10 \mathrm{~cm}$ diameter non-tender firm palpable abdominal mass, which on computed tomography was diagnosed to be a $12.2 \times 10.3 \times 8.0 \mathrm{~cm}$ heterogeneously enhancing adrenal mass having absolute and relative contrast washout values suggestive of malignancy. The tumor was hormonally inactive. Left adrenalectomy was done. Resected specimen weighed $91 \mathrm{~g}$ measuring $12 \times 10 \times 8.0$ $\mathrm{cm}$. Histopathology revealed a well-circumscribed and encapsulated benign spindle cell arranged in fascicles and whorls confirming leiomyoma. In the last evaluation 6 months after surgery, there was no evidence of tumor recurrence. This is the largest adrenal leiomyoma ever reported. Leiomyomas have a varied age of presentation (2 72 years, median: 38 ) with female preponderance. They are usually unilateral and hormonally inactive. Human immunodeficiency virus and Epstein-Barr virus infections have been observed in $44.44 \%$ and $16.67 \%$ of cases respectively. However, direct link between immunodeficiency and adrenal leiomyoma has not been established. Adrenal leiomyoma can present as huge abdominal masses clinically mimicking malignancy, and radiologic investigations can also be misleading. Adrenal leiomyoma should always be considered in the
\end{abstract}

Manuscript accepted for publication October 26, 2015

aDepartment of Endocrinology, Kalinga Institute of Medical Sciences (KIMS), Bhubaneswar, India

${ }^{b}$ Department of Endocrinology, Post Graduate Institute of Medical Education \& Research (PGIMER) and Dr Ram Manohar Lohia (RML) Hospital, New Delhi, India

${ }^{\mathrm{c}}$ Department of Pathology, Kalinga Institute of Medical Sciences (KIMS), Bhubaneswar, India

${ }^{\mathrm{d} D e p a r t m e n t}$ of Oncosurgery, Kalinga Institute of Medical Sciences (KIMS), Bhubaneswar, India

${ }^{\mathrm{e}}$ Corresponding Author: Deep Dutta, Department of Endocrinology, Post Graduate Institute of Medical Education \& Research (PGIMER) and Dr Ram Manohar Lohia (RML) Hospital, New Delhi 110001, India.

Email: deepdutta2000@yahoo.com

doi: http://dx.doi.org/10.14740/jem318w differential diagnosis of huge unilateral non-functional incidentally detected adrenal lesions.

Keywords: Adrenal leiomyoma; Carcinoma; Malignancy; Neoplasm; Incidentaloma

\section{Introduction}

Leiomyomas are benign tumors arising from smooth muscle cells lining various organs in the body, most commonly observed in uterus followed by the gastrointestinal tract $[1,2]$. Leiomyoma arising from adrenals are extremely rare with less than 20 cases reported in medical literature, to the best of our knowledge [1-18]. Adrenal leiomyomas are clinically asymptomatic, hence they usually present as incidentalomas $[3,16,17]$. Adrenal incidentalomas have been documented in approximately $1 \%$ of all abdominal computed tomography (CT) scans [19-21]. Leiomyomas, though benign lesions, may frequently be confused with a malignant tumor, both clinically and on imaging studies, especially when they occur at atypical sites like the adrenals. This report presents a middle-aged lady with cachetic symptoms, presenting with a palpable huge abdominal mass, which was subsequently diagnosed to be adrenal leiomyoma. A subsequent review of all available literature on adrenal leiomyoma was also done.

References for review of literature on adrenal leiomyoma were identified through searches of PubMed, Medline, and Embase for articles published to September 2015 using the terms "adrenal leiomyoma" (MeSH Terms) OR "adrenal leiomyoma" (all fields). The reference lists of the articles thus identified were also searched. The search was not restricted to English-language literature.

\section{Case Report}

A 42-year-old lady presented with loss of appetite and weight (12 kg in 4 months) along with heaviness and dragging sensation on left side of her abdomen of 6-month duration. Examination was significant for body mass index of $17.2 \mathrm{~kg} / \mathrm{m}^{2}$, normal blood pressure and a visible abdominal swelling which on palpation revealed a $10 \mathrm{~cm}$ diameter non-tender firm abdomi- 


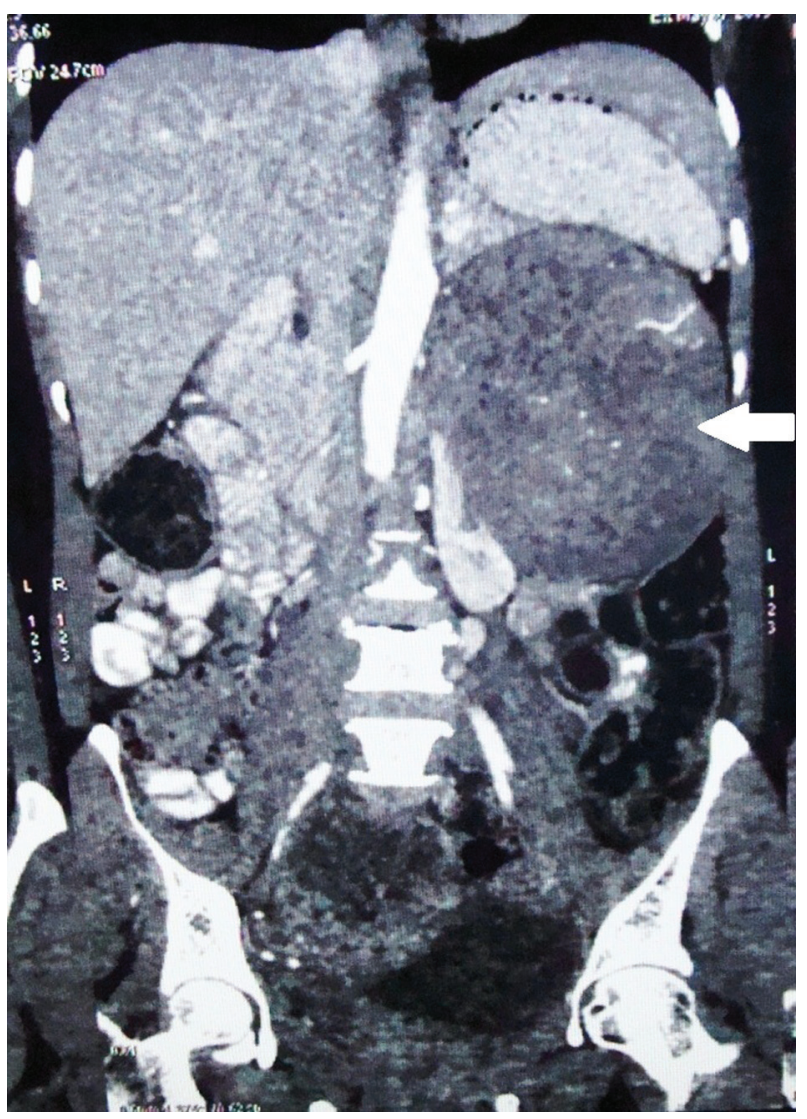

Figure 1. Contrast-enhanced computed tomography (CT) of abdomen showing a large left-sided adrenal mass measuring $12.2 \times 10.3 \times 8.0$ $\mathrm{cm}$. nal mass with smooth and regular margin and moving with respiration. Screening ultrasonography revealed a retroperitoneal suprarenal mass suggestive of adrenal origin. Clinical stigmata of Cushing's syndrome, Addison's disease or pheochromocytoma were absent. Biochemical investigations were significant for normal electrolytes, normal morning 9 am cortisol $(17 \mu \mathrm{g} /$ $\mathrm{dL})$, post-overnight dexamethasone suppressed morning cortisol $(1.6 \mu \mathrm{g} / \mathrm{dL})$ and normal urinary metanephrines $(12.2 \mu \mathrm{g}$ in $24 \mathrm{~h})$. Plasma renin activity and serum aldosterone were not evaluated as the patient did not have hypertension. Serologic test for human immunodeficiency virus and Epstein-Barr virus (EBV) was negative. CT of abdomen revealed a large $(12.2 \times$ $10.3 \times 8.0 \mathrm{~cm})$ heterogeneously enhancing adrenal mass with regular margins abutting the upper pole of the left kidney, with areas of necrosis (Fig. 1). There was no internal calcification. The pre-contrast, immediate post-contrast and delayed postcontrast Hounsfield unit (HU) values of the adrenal mass were 65, 96 and 84, respectively. Hence the absolute and relative contrast washout values were $38.7 \%$ ( $>60 \%$ suggestive of benign lesion) and $12.5 \%$ ( $>40 \%$ suggestive of benign lesion) respectively, suggestive of malignant lesion [22]. Hence left adrenalectomy was done. The resected specimen weighed $91 \mathrm{~g}$ and measured $12 \times 10 \times 8.0 \mathrm{~cm}$ (Fig. 2A, B). Macroscopically, the tissue had a white appearance and there was no bleeding, ulceration or necrosis (Fig. 2A, B). Histopathology revealed a well-circumscribed and encapsulated benign spindle cell arranged in fascicles and whorls. There were few areas of hyaline degeneration. No features of mitosis or pleomorphism were present (Fig. 3A, B). Recovery was uneventful and in the last evaluation 6 months after the surgery, the patient was doing well, clinically asymptomatic, and had regained $8 \mathrm{~kg}$ body weight.

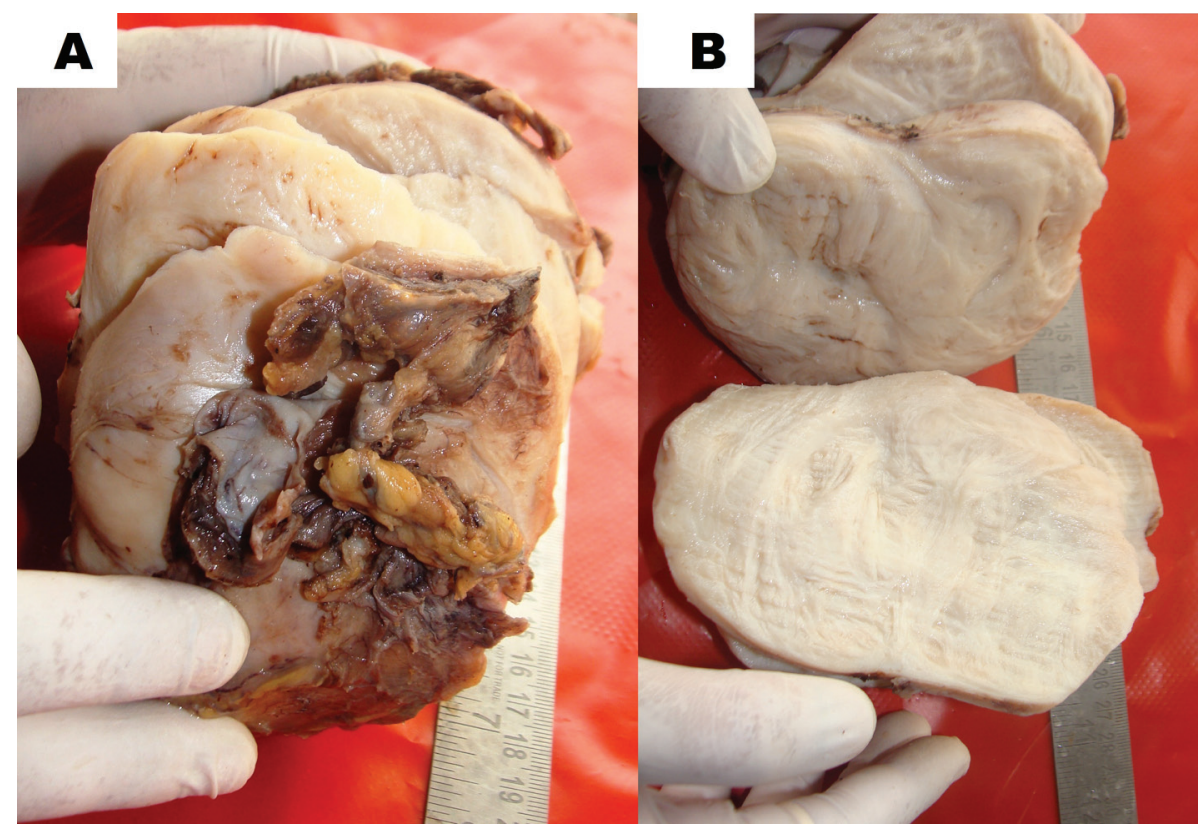

Figure 2. (A) Post-surgical resected specimen showing well-encapsulated adrenal mass. (B) Resected specimen's cut surface showing typical whorling appearance classical of leiomyoma. 


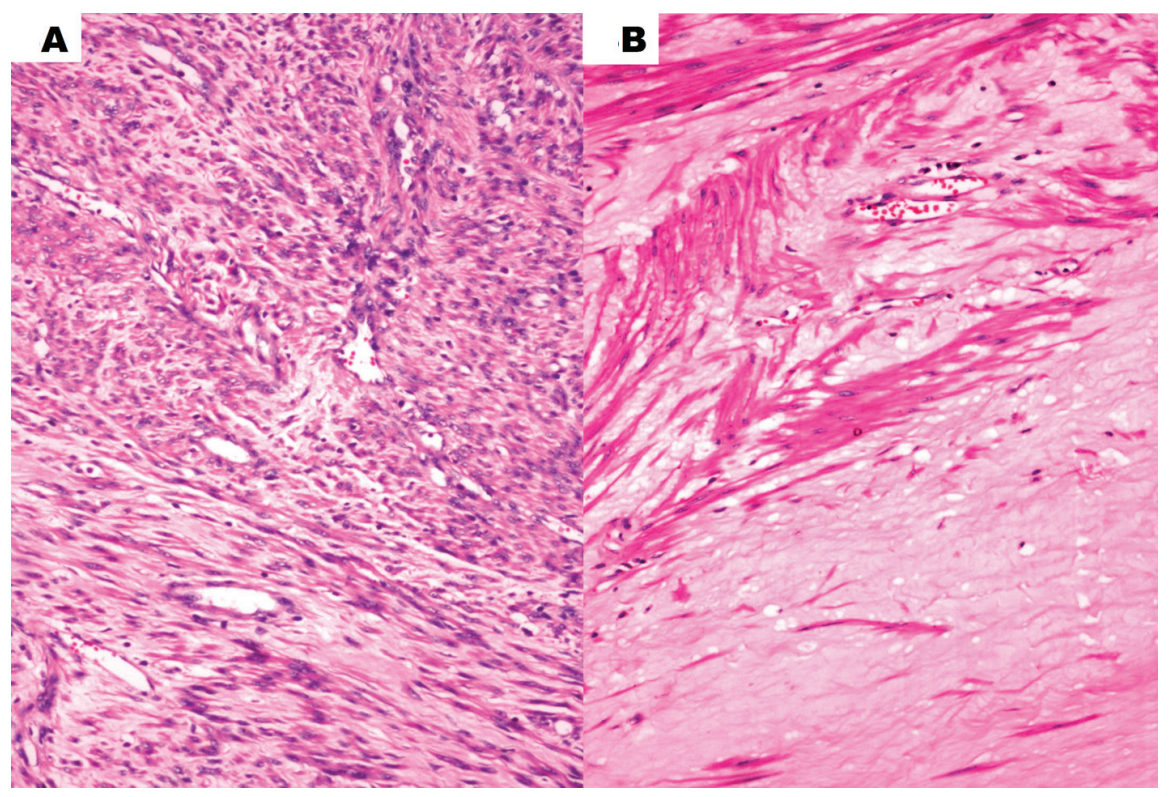

Figure 3. (A) Histopathology of the resected adrenal tumor showing bundles of smooth muscle cells diagnostic of leiomyoma (hematoxylin \& eosin stain, $\times 200$ ). (B) Higher magnification showing hyaline degeneration within the leiomyoma (hematoxylin $\&$ eosin stain, $\times 400)$.

Table 1. Profile of Patients With Adrenal Leiomyoma Reported in Literature as Compared to the Present Report

\begin{tabular}{|c|c|c|c|c|c|c|c|}
\hline \multirow{2}{*}{ S. No. } & \multirow{2}{*}{ Reported cases } & \multirow{2}{*}{ Age (years) } & \multirow{2}{*}{ Sex } & \multicolumn{2}{|c|}{ Tumor characteristics } & \multirow{2}{*}{ HIV status } & \multirow{2}{*}{ EBV status } \\
\hline & & & & Functional ${ }^{\mathrm{a}}$ & Size $(\mathbf{m m})^{\mathbf{b}}$ & & \\
\hline 1 & Lin et al [1] & 31 & Female & No & $110 \times 90 \times 70$ & Positive & Negative \\
\hline 2 & Nishida et al [2] & 48 & Female & No & $55 \times 45 \times 50$ & Negative & Negative \\
\hline 3 & Alteer et al [3] & 40 & Male & No & $49 \times 55 \times 60$ & Positive & Positive \\
\hline 4 & Chang et al [4] & 53 & Female & No & $55 \times 45 \times 35$ & Positive & Negative \\
\hline 5 & Radin et al [5] & 28 & Female & NA & 30 & Positive & Negative \\
\hline 6 & Jimenez-Heffernan et al [6] & 2 & Male & NA & $70 \times 50 \times 50$ & Positive & Positive \\
\hline 7 & Mouchet et al [7] & 10 & Female & NA & $50 \times 40 \times 30$ & Negative & Negative \\
\hline 8 & Gibbs et al [8] & 49 & Female & No & $30 \times 35 \times 20$ & Negative & Negative \\
\hline 9 & Jacobs et al [9] & 65 & Female & No & $50 \times 31 \times 42$ & Negative & Negative \\
\hline 10 & Parola et al [10] & 35 & Female & NA & 35 & Positive & Negative \\
\hline 11 & Goldman et al [11] & 72 & Male & No & $90 \times 70 \times 60$ & Negative & Negative \\
\hline 12 & Dahan et al [12] & 32 & Male & No & 30 & Positive & Negative \\
\hline 13 & Rosenfeld et al [13] & 11 & Female & NA & $\mathrm{L}: 50, \mathrm{R}: 30$ & Positive & Negative \\
\hline 14 & Demirel et al [14] & 15 & Male & No & $\begin{array}{l}\mathrm{L}: 40 \times 50 \times 35 \\
\mathrm{R}: 80 \times 50 \times 30\end{array}$ & Negative & Negative \\
\hline 15 & Chao et al [15] & 40 & Female & No & NA & Negative & Negative \\
\hline 16 & Jurczak et al. [16] & 56 & Male & No & 72 & Negative & Negative \\
\hline 17 & Al-Masri et al [17] & 38 & Female & NA & NA & Negative & Negative \\
\hline 18 & Parelkar et al [18] & 11 & Female & No* & $\begin{array}{l}\mathrm{L}: 43 \times 35 \times 30 \\
\mathrm{R}: 90 \times 70 \times 40\end{array}$ & Negative & Positive \\
\hline 19 & Present report & 42 & Female & No & $120 \times 100 \times 80$ & Negative & Negative \\
\hline
\end{tabular}

${ }^{a}$ Functional refers to the functional status of the tumor, viz. its ability to secrete one or more hormones either from the adrenal cortex or medulla. ${ }^{b}$ For tumors with single value, it represents the largest diameter. *The 24-h urinary vanillylmandelic acid was normal. Plasma normetanephrine was increased, which is commonly known to be false-positive. NA: not available; HIV: human immunodeficiency virus; EBV: Epstein-Barr virus; L: left; R: right. 


\section{Discussion}

Leiomyomas have been most commonly reported from the uterus followed by the gastrointestinal tract [1-3]. Leiomyomas are extremely rare tumors of the adrenal gland [1-5]. They are classically asymptomatic, and usually detected incidentally when abdominal imaging is done for some other cause (incidentaloma), and diagnosis is usually on histopathologic examination of the resected specimen. The cases reported in literature have been summarized in Table 1 [1-18]. Leiomyomas have a varied age of presentation ranging from 2 to 72 years (median: 38 years) with a female preponderance (12 out of 18; $66.67 \%$ ) (Table 1). Adrenal leiomyomas reported in literature were typically unilateral, solitary and have a varied size ranging from 30 to $110 \mathrm{~mm}$ in diameter (median largest diameter: $50 \mathrm{~mm}$ ) (Table 1). Bilateral adrenal leiomyoma are even rarer, predominantly reported in children $[14,18]$. We would like to highlight that the leiomyoma in our patient is the largest adrenal leiomyoma ever reported in indexed medical literature. Interestingly immunodeficiency has been linked to adrenal leiomyoma both in the pediatric and adult age groups. HIV infection or acquired immune deficiency syndrome (AIDS) has been observed in eight out of $18(44.44 \%)$ cases reported [1-18]. There are few reports of adrenal leiomyoma occurring in patients with latent EBV infection (three out of 18; 16.67\%) $[3,6,18]$. However, direct link between immunodeficiency and adrenal leiomyoma has not been established. It has been postulated that HIV infection may promote smooth muscle tumors through a direct or indirect oncogenic stimulatory effect [10]. HIV is well known to be associated with lymphomas and Kaposi sarcoma [10]. All the adrenal leiomyomas reported till date (including our patient) were biochemically non-functional, viz. did not have any evidence of one or more hormone production either from adrenal cortex or medulla.

It is important to highlight that in contrast to previous reports, our patient did not have immunodeficiency, and was clinically symptomatic at the time of presentation. The radiologic features on CT in our patient mimicking malignancy have also been observed previously $[1,12,18]$. This report intends to highlight that adrenal leiomyoma is a rare benign non-functional adrenal neoplasm with good clinical outcomes following surgical excision. Adrenal leiomyoma can present as huge abdominal masses mimicking malignancy, and even radiologic investigations can be misleading in terms of differentiating this benign lesion from malignant adrenal lesions like adrenal carcinoma or pheochromocytoma. Adrenal leiomyoma should always be considered in the differential diagnosis of huge unilateral non-functional incidentally detected adrenal lesions.

\section{Conflict of Interests}

None.

\section{References}

1. Lin J, Wasco MJ, Korobkin M, Doherty G, Giordano TJ:
Leiomyoma of the adrenal gland presenting as a non-functioning adrenal incidentaloma: case report and review of the literature. Endocrine pathology 2007, 18(4):239-243.

2. Nishida S, Tanimura A, Takasaki S, Nagaoka S, Fukueda M, Ikeda S, Matsuo K, Akao M, Tokunaga M: Surgically resected adrenal leiomyoma: report of a case. Surgery today $1995,25(5): 455-457$.

3. Alteer M, Ascott-Evans BH, Conradie M. Leiomyoma: a rare cause of adrenal incidentaloma. JEMDSA. 2013;18:71-74.

4. Chang TH, Lee YC, Liu CC, Huang CH, Wu WJ: Adrenal leiomyoma treated by hand-assisted laparoscopic adrenalectomy: a case report. The Kaohsiung journal of medical sciences 2006, 22(11):575-579.

5. Radin DR, Kiyabu M: Multiple smooth-muscle tumors of the colon and adrenal gland in an adult with AIDS. AJR American journal of roentgenology 1992, 159(3):545546.

6. Jimenez-Heffernan JA, Hardisson D, Palacios J, GarciaViera M, Gamallo C, Nistal M: Adrenal gland leiomyoma in a child with acquired immunodeficiency syndrome. Pediatric pathology \& laboratory medicine : journal of the Society for Pediatric Pathology, affiliated with the International Paediatric Pathology Association 1995, 15(6):923-929.

7. Mouchet F, Ninane J, Gosseye S, Verellen C, Bonnier C, Evrard P, Vermylen C, Scheiff JM, Cornu G: Leiomyoma of the suprarenal gland in a child with ataxia-telangiectasia. Pediatric hematology and oncology 1991, 8(3):235241.

8. Gibbs KE, White A, Kaleya R: Laparoscopic management of an adrenal leiomyoma in an AIDS patient. A case report and review of the literature. JSLS : Journal of the Society of Laparoendoscopic Surgeons / Society of Laparoendoscopic Surgeons 2005, 9(3):345-348.

9. Jacobs IA, Kagan SA: Adrenal leiomyoma: a case report and review of the literature. Journal of surgical oncology 1998, 69(2):111-112.

10. Parola P, Petit N, Azzedine A, Dhiver C, Gastaut JA: Symptomatic leiomyoma of the adrenal gland in a woman with AIDS. AIDS 1996, 10(3):340-341.

11. Goldman RL, Brodey PA: Symptomatic leiomyoma of the adrenal. Clinical imaging 1994, 18(4):277-278.

12. Dahan H, Beges C, Weiss L, Abitbol M, Ledreff O, Teman G, Belattar K, Rymer R: Leiomyoma of the adrenal gland in a patient with AIDS. Abdominal imaging 1994, 19(3):259-261.

13. Rosenfeld DL, Girgis WS, Underberg-Davis SJ: Bilateral smooth-muscle tumors of the adrenals in a child with AIDS. Pediatric radiology 1999, 29(5):376-378.

14. Demirel S, Erk O, Akkaya V, Tunaci A, Tanakol R, Terzioglu T, Demiryont M, Kadioglu A, Senkal O: Multiple vascular leiomyomas involving bilateral adrenal glands, spleen, and epicardium, associated with bilateral testicular microlithiasis and empty sella turcica. Journal of pediatric surgery 1997, 32(9):1365-1367.

15. Chao CS, Zhou ZG, Liao EY: [The diagnosis and management of adrenal "incidentaloma"]. Zhonghua nei ke za zhi [Chinese journal of internal medicine] 1994, 33(6):395- 
397.

16. Jurczak F, Hamy A, Paineau J, Courant O, Visset J: [An unusual "incidentaloma": adrenal leiomyoma]. Journal de chirurgie 1994, 131(8-9):391.

17. Al-Masri AA, Aladily TN, Younes NA: Unusual association between adrenal leiomyoma and autoimmune disease. Saudi medical journal 2010, 31(2):199-201.

18. Parelkar SV, Sampat NP, Sanghvi BV, Joshi PB, Sahoo SK, Patel JL, Oak SN: Case report of bilateral adrenal leiomyoma with review of literature. Pediatric surgery international 2013, 29(6):655-658.

19. Bovio S, Cataldi A, Reimondo G, Sperone P, Novello S, Berruti A, Borasio P, Fava C, Dogliotti L, Scagliotti GV et al: Prevalence of adrenal incidentaloma in a contemporary computerized tomography series. Journal of endocrinological investigation 2006, 29(4):298-302.

20. Lack EE. Smooth muscle neoplasms. In: Lack EE, ed. AFIP Atlas of Tumor Pathology, Tumors of the Adrenal Glands and ExtraadrenalParaganglia. Fourth Series. Fascicle 8. Maryland: Silver Spring. 2007;201.

21. Young WF, Jr.: Clinical practice. The incidentally discovered adrenal mass. The New England journal of medicine 2007, 356(6):601-610.

22. Allen BC, Francis IR: Adrenal Imaging and Intervention. Radiologic clinics of North America 2015, 53(5):10211035 . 